\title{
Conductivity Percolation on a Square Lattice with Two Different Sizes of Particles
}

\author{
Kazuhito Shida, Ryoji Sahara, MN Tripathi, Hiroshi Mizuseki and Yoshiyuki Kawazoe \\ Institute for Materials Research (IMR), Tohoku University, Sendai 980-8577, Japan
}

Electric conductance of percolation clusters is calculated by means of the random circuit approximation, under a binary distribution in the size of the conducting particles. Although we have already investigated the same size distribution model in terms of the percolation threshold, the electric transport conductance of this model is never reported before. The size distribution of conducting particles induces a clear difference in the ensemble of random circuit. However, the observed critical behavior and critical exponents are closely matched to those reported for monodisperse cases. [doi:10.2320/matertrans.M2009202]

(Received June 22, 2009; Accepted September 28, 2009; Published November 11, 2009)

Keywords: thin film conductor, percolation model, critical phenomenon, granular material

\section{Introduction}

The percolation model ${ }^{1)}$ is a simple and powerful theoretical tool which plays an important role in explaining various physical phenomena related to properties of disordered systems. There are two basic classes of percolation models: the site-percolation and the bond-percolation models. A site-percolation model is defined on a lattice, whose sites are randomly occupied at probability $p$. At a critical threshold $p_{\mathrm{c}}$ of the site occupation probability, a phase transition regarding interconnectivity occurs from an nonpercolating phase to a percolating phase, where at least one passage through occupied sites is available between almost all pairs of occupied sites.

Alternatively, a bond-percolation model can be defined on the same lattice by randomizing the existence of lattice edges (they are connected at probability $p$ ). The behavior of the criticality shows a strong "universality" (independence from the type of the lattice). The strength of such percolation models derives from their abstract nature: detailed characteristics of the passage like width and length are completely omitted from the model. However, these characteristics should be reintroduced to the model when percolation models are considered in a model with even a modest level of physical details.

For example, Monte Carlo simulations of transport media have been performed for a continuum "Swiss-cheese percolation model". Detailed studies have revealed that when void-size distribution, ${ }^{2-6)}$ that is related to the distribution of width, is introduced to this model, the threshold $p_{\mathrm{c}}$ depends on this void-size distribution. The universality of this model could be violated, although statistical errors involved in previous calculations did not allow the full confirmation of this phenomenon. On the other hand, a good example of a percolation model with the idea of passage length is the mean first-passage-time percolation model, ${ }^{7)}$ where the passage "time" (in other words, the length) is associated with every connected pair of sites in the system. Then, the time-course of the propagation of particles (representing, for example, the spread of a contagious disease) through the network is considered.
In a previous work, ${ }^{8,9)}$ we proposed and examined a new type of 2D site-percolation model where two different sizes of particles, i.e., particles $\mathrm{A}$ and $\mathrm{B}$, are present on a square lattice. In the model, the smaller particle (particle A) can occupy one site of the lattice just as in the ordinary model, whereas the larger particle (particle B) can occupy multiple sites. Lattice sites are occupied, either by particle A or by a part of particle $\mathrm{B}$, with probability $p$. Here, the overall density of the conducting site, $p$ is the sum of the site occupation probabilities $p_{\mathrm{A}}$ and $p_{\mathrm{B}}$, which are the site occupation probabilities of particles $\mathrm{A}$ and $\mathrm{B}$, respectively:

$$
p=p_{\mathrm{A}}+p_{\mathrm{B}} .
$$

In this paper, we confine ourselves to the simplest condition of $p_{\mathrm{A}}=p_{\mathrm{B}}$.

Despite the clear difference from the standard model in the particle size distribution, the percolation threshold, $p_{\mathrm{c}}$, did not exhibit a large deviation from that of the standard percolation model in our previous work. Other authors reported different $p_{\mathrm{c}}$ for $2 \mathrm{D}$ percolation with uniform and variable particle sizes, although the increase caused by the variable particle sizes is in an order of 0.01 . When $p_{\mathrm{A}}$ is not equal to $p_{\mathrm{B}}$, stronger dependence of $p_{\mathrm{c}}$ on the particle size distribution is reported to be possible. ${ }^{2,10)}$

In this paper, we employ the same extended percolation model proposed before, but calculate the electric transport phenomenon for the percolation cluster. Electric conductance of a percolation cluster is dependent not only on the existence of a large cluster, but also on the "width" of the percolating cluster perpendicular to the direction of the current. The electric conductance is theoretically interesting because it may have a different exponent from the normal percolation transition. ${ }^{1)}$ On the other hand, the electric conductance has a great importance associated with real material-design applications where the conductance, the cost, and other characteristics of the target material must be balanced. This is particularly true in some applications where conducting materials must simultaneously satisfy special characteristics other than the electronic conductance, because such materials tend to be less abundant and, as a consequence, more expensive. A good example of such a special characteristics 
is the optical transparency; Transparent conducting films are materials of great technological importance because they are necessary in making flat panel displays or solar cells. Regarding low-cost production of such films, deposition methods based on nano-scale particles ${ }^{11-15)}$ have been a focus of intense interest. The particle based film production is strongly relevant to the 2D percolation model investigated in this report. The following is, to the authors' best knowledge, the first report on the electric conductance of a model of 2D granular materials with particle size distributions.

\section{Methods}

The electric conductance of percolating cluster ${ }^{16)}$ was calculated considering a $2 \mathrm{D}$ random resistor network on a square lattice where the value of resistance is $R$ between two conducting sites, and 0 elsewhere. As explained above, these conducting sites may belong to particles with different sizes (see Fig. 1).

The largest connecting cluster of conducting sites that "bridge" the left and right edges of the lattice is calculated by a standard cluster algorithm. Then the Kirchhoff equations are solved on the resister network formed by the largest cluster of conducting sites by means of a simple relaxation method to calculate the total current flowing into the left edge of the system, while the voltage between the right and left edges of the system is kept constant. Since a 2D system is considered, the whole current between the edges can be regarded as the effective conductance of the system. When the calculation does not converge in 500000 steps, the cluster is simply omitted from the calculation, such that the effect of critical slowing down on the relaxation method is canceled. If there is no bridging cluster, the conductance is obviously 0 and the Kirchhoff equations are not solved. These clusters with 0 conductance are also omitted from the calculation.

The lattice sizes, $L$, used in the simulations are 100, 200, 300,400 , and 500 for calculation of the critical exponent. Basically, the range of $0.580 \sim 0.628$ is scanned as the value of $p$, and the critical point is decided by the accordance of the result to eq. 5 .

Since we are also interested in the general level of the conductance, bigger lattice sizes, such as $1000 \times 1000$ and $2000 \times 2000$, are also used with $p$ values outside the critical region. Typically, 1000 8000 randomly generated configurations are calculated, depending on the size of the lattice.

For a random 2D register network at the critical density, which is close to the critical density of the conventional bond-percolation model, $p=0.5$, it is reported ${ }^{17)}$ that the correlation length $\xi$ diverges as

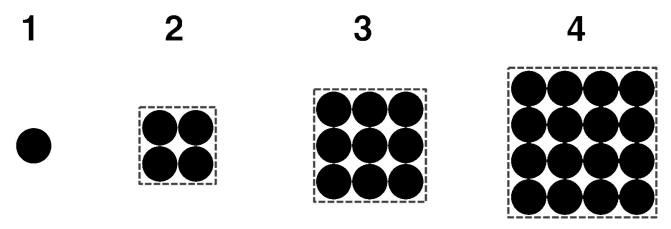

Fig. 1 In this work, these particle designs are used to simulate the size variations of the conducting materials. The filled circles are individual conducting sites. The multiple sites surrounded by a dashed line are regarded as a particle with larger sizes.

$$
\xi \propto\left(p-p_{\mathrm{c}}\right)^{-v}
$$

where $v$ is a critical exponent, the value of which is independent of the details of the lattice.

The conductivity, denoted by $\sigma$, follows the relationship

$$
\sigma \propto\left(p-p_{\mathrm{c}}\right)^{\mu}
$$

where $\mu$ is an another exponent.

Considering the finite scaling hypothesis, such as

$$
\sigma(L, \xi)=\xi^{-\mu / v} S(L / \xi)
$$

where $S()$ is an appropriate scaling function, we can derive that

$$
\sigma \propto L^{-\mu / v}
$$

for finite $L$, in the close vicinity of the critical point (diverging $\xi$ ). When the conductivity is plotted against the system size in a double-log manner, the criticality of the system is manifested by a linear plot and its slope has the value of $-\mu / \nu$.

\section{Results}

Our result in Fig. 2 shows that the effective conductance of our model follows the same relationship observed for the standard random network model, but with evidently different values of critical point. This difference can be attributed to the statistical nature of the network: in previous works on the random register network, resisters are placed on randomly selected edges of the lattice whereas in our work the 2D register network is generated as a representation of the conductance in granular materials (resistance is $R$ between two conducting sites and 0 elsewhere).

The difference between these two types of 2D register networks is exemplified by the number of local resister configurations prohibited in our model, for example the one shown in Fig. 3. Now it is easy to see that these two different ensembles of random resister networks are "conductivity versions" of the bond-percolation model and site-percolation model (this work), respectively.

It should be also noted that the conductivity version of the site-percolation model we investigate in this paper may

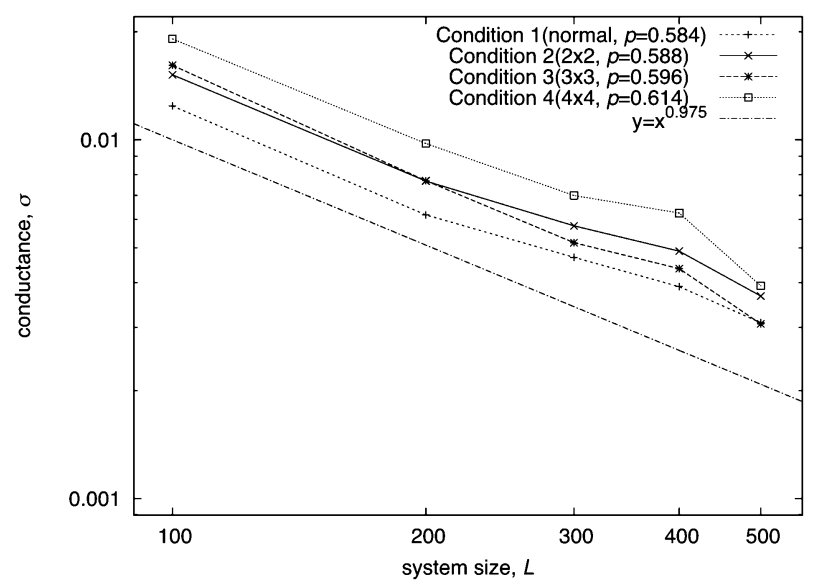

Fig. 2 Lattice size versus conductivity plots. The power-law dependence is clear, showing finite-size scaling in the conductivity. 


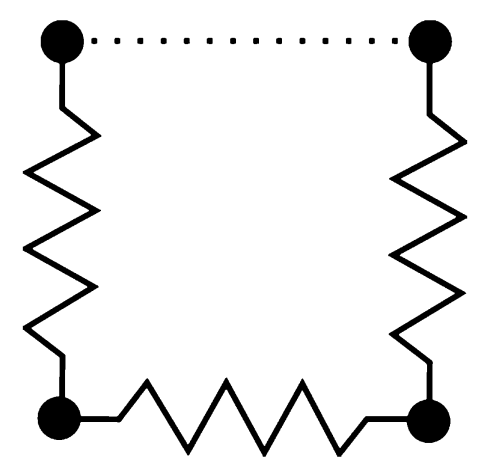

Fig. 3 Unlike the random register network investigated before, our model prohibits this register configuration. Because all neighboring conducting sites (filled circles) should be always connected via the registers (wavy lines) in our model, the absence of connection (shown by the dashed line at top) is prohibited. Note that such an absence is allowed in original from of the random register network model. This condition defines "conductivity version" of the site-percolation model.

Table 1 Observed value of critical points for different degree of particle size distributions.

\begin{tabular}{lcccc}
\hline Particle type & $1(1 \times 1)$ & $2(2 \times 2)$ & $3(3 \times 3)$ & $4(4 \times 4)$ \\
\hline Critical point & 0.584 & 0.588 & 0.596 & 0.614 \\
\hline
\end{tabular}

be a better model of composite conductive material, because it is rather unnatural to include the configuration shown in Fig. 3 to investigate the conducting behavior of the granular materials.

The following is, to the authors' best knowledge, the first report on the critical behavior of the "conductivity version" of the site percolation model. The conductivity versus lattice size plot shows the best linearity near $p=0.588$. This value is clearly different from the critical point of the conductivity version of bond-percolation, $p=0.500$, and very close to that of the ordinary version of site-percolation $(p=0.593)$ as anticipated. This clearly demonstrates the difference between conduction models corresponding to bond- and site-percolation models. The value of the critical exponent estimated by the slopes in Fig. 2 is closely matched to the value of critical exponents reported for "conductivity versions" of bond-percolation $(0.975)$ for $2 \mathrm{D},{ }^{18)}$ presenting a proof of that this model belongs to the universality class of ordinary bond-percolation.

Next, we introduced a particle size distribution by means of large conducting particles in the model, with the result shown in Fig. 2. It seems that as the size of the large particle increases, the best linearity is observed at higher densities. A similar magnitude of increase of critical density has already been observed in our previous works for contact percolations. The critical points are tabulated in Table 1, and we have no experimental or theoretical results to compare to this value.

The general (not limited to the critical region) behavior of the effective conductance is shown in Fig. 4. The large conducting particles are expected to be a kind of "bridge" in the random register network; as a result, the overall conductance is expected to increase by the introduction of large particles. Contrary to this intuition, this figure shows that including large conducting particles in the system is not an effective way to increase the overall conductivity.

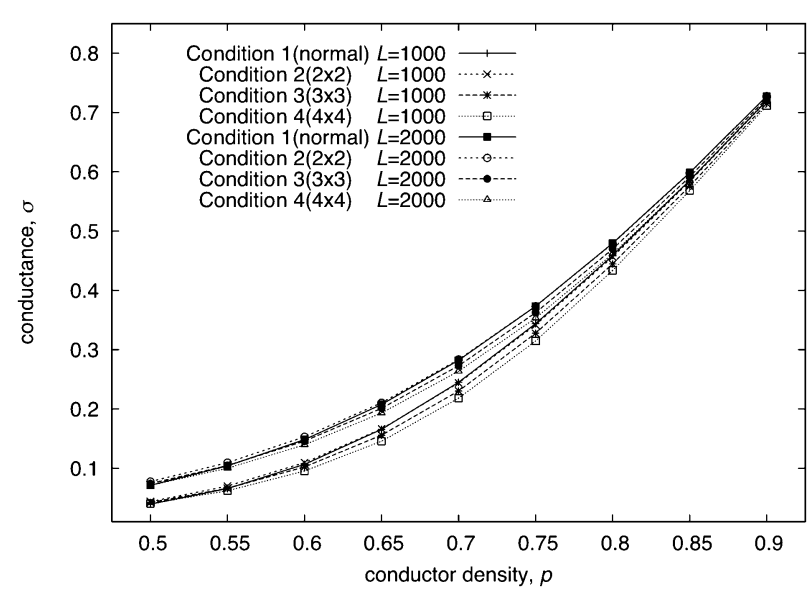

Fig. 4 General behavior of the conductance. The irregularity in particle sizes has a weak and negative effect on the global conductance of the system.

Since the overall density, $p$, of conducting sites is not changed by the introduction of particle $\mathrm{B}$, we are modulating only the spatial distribution of conducting materials. That is, even if conduction inside and around B particles can be improved, the conductor density anywhere else in the system is reduced. At the same time, the conductance is a rather steep increasing function of conducting particle density even when the system is far from the percolation threshold. The combination of these two factors seems to be able to address the difficulties of improving the system conduction by "bridging": Improving conductance at some particular portion of the system does not give rise to the improvement of the conductance of entire system. In other words, this result tells us the possibility of negatively controlling the conductance of composite materials by modulating the spatial distribution of conducting materials.

\section{Discussion}

The conductivity percolation on random register networks has been investigated for a long time, but similar conductance percolation in a "site-version" of the same problem has been neglected. In this short paper, this problem is defined and investigated by a large-scale conductance simulation. The main reason of considering the conductance in this model is that it is strongly connected to the technologically important applications of the nano particle based thin films, such as flat panel displays. The conductance shows a power-law behavior just like the conventional random register models; however, at a different critical point. The critical point, which can be defined as the critical density of conducting particles, coincides with that of the ordinary site-percolation threshold on the same lattice. The critical exponent of this new type of percolation phenomena is for the first time estimated and reported.

Also, the effect of particle size distribution is investigated on the same lattice; the effect of the large particles closely resembles their effect on the conventional sitepercolation process. That is, the introduction of particle size distribution caused very small changes in the critical conductance and the exponents in the critical behavior, but 
these changes are not likely to be used to decrease the critical density. In general, distributions of different-size conducting particles reduce the conductivity per given amount of conducting materials. This result will contribute to the better material designs related to transparent thin films. Of course, we must extend this research to conditions with general values of $p_{\mathrm{A}}$ and $p_{\mathrm{B}}$. However, this needs much more computational time and more elaboration in computational methods because it is ideal to integrate other types of irregularities in the particle design, including the particle shape difference ${ }^{19)}$ and size distribution of insulator particles. $^{20)}$

\section{Acknowledgement}

This work was supported by the Ministry of Economy, Trade and Industry (METI) under "Development for reduction of Indium in a transparent electrode ITO". The authors gratefully acknowledge the Center for Computational Materials Science at the IMR of Tohoku University.

\section{REFERENCES}

1) D. Stauffer and A. Aharony: Introduction to Percolation Theory, (Taylor and Francis 1992).

2) M. K. Phani and D. Dhar: J. Phys. A 17 (1984) L645.
3) B. Lorenz, I. Orgzall and H.-O. Heuer: J. Phys. A: Mathematical and General 26 (1993) 4711-4722.

4) B. Lorenz and I. Orgzall: Philos. Mag. B 71 (1995) 407-412.

5) S. C. vanderMarck: Phys. Rev. Lett. 77 (1996) 1785-1788.

6) A. Klemm, H. P. Muller and R. Kimmich: Phys. Rev. E 55 (1997) 4413-4422.

7) R. T. Smythe and J. C. Wierman: First-passage percolation on the square lattice, (Springer 1978).

8) R. Sahara, H. Mizuseki, K. Ohno and Y. Kawazoe: Mater. Trans. JIM 40 (1999) 1314-1318.

9) R. Sahara, H. Mizuseki, K. Ohno and Y. Kawazoe: J. Phys. Soc. Japan 68 (1999) 3755-3758.

10) D. Dhar: Phys. A 242 (1997) 341-346.

11) R. B. H. Tahar, T. Ban, Y. Ohya and Y. Takahashi: J. Appl. Phys. 82 (1997) 865-870.

12) K. Nishio, S. Miyake, T. Sei, Y. Watanabe and T. Tsuchiya: J. Mater. Sci. 31 (1996) 3651-3656.

13) A. Tsunashima, H. Yoshimizu, K. Kodaira, S. Shimada and T. Matsushita: J. Mater. Sci. 21 (1986) 2731-2734.

14) T. D. Senguttuvan and L. K. Malhotra: Thin Solid Films 289 (1996) 22-28.

15) C. Terrier, J. P. Chatelon and J. A. Roger: Thin Solid Films 295 (1997) 95-100.

16) S. Kirkpatrick: Rev. Modern Phys. 45 (1973) 574-588.

17) M. Sahimi, B. D. Hughes, L. E. Scriven and H. T. Davis: J. Phys. C 16 (1983) L521-L527.

18) J. M. Normand, H. J. Herrmann and M. Hajjar: J. Statistical Phys. 52 (1988) 441-446.

19) D. He and N. N. Ekere: J. Phys. D 37 (2004) 1848-1852.

20) M. Ambrozic, A. Dakskobler and M. Valant: Europ. Phys. J. Appl. Phys. 30 (2005) 23-31. 